\title{
Implementation of Statistical Process Control: Evaluating the Mechanical Performance of a Candidate Silicone Elastomer Docking Seal
}

\author{
Heather A. Oravec ${ }^{1}$ and Christopher C. Daniels ${ }^{2}$ \\ The University of Akron, Akron, Ohio, 44325-3901, USA
}

\begin{abstract}
The National Aeronautics and Space Administration has been developing a novel docking system to meet the requirements of future exploration missions to low-Earth orbit and beyond. A dynamic gas pressure seal is located at the main interface between the active and passive mating components of the new docking system. This seal is designed to operate in the harsh space environment, but is also to perform within strict loading requirements while maintaining an acceptable level of leak rate. In this study, a candidate silicone elastomer seal was designed, and multiple subscale test articles were manufactured for evaluation purposes. The force required to fully compress each test article at room temperature was quantified and found to be below the maximum allowable load for the docking system. However, a significant amount of scatter was observed in the test results. Due to the stochastic nature of the mechanical performance of this candidate docking seal, a statistical process control technique was implemented to isolate unusual compression behavior from typical mechanical performance. The results of this statistical analysis indicated a lack of process control, suggesting a variation in the manufacturing phase of the process. Further investigation revealed that changes in the manufacturing molding process had occurred which may have influenced the mechanical performance of the seal. This knowledge improves the chance of this and future space seals to satisfy or exceed design specifications.
\end{abstract}

\section{Nomenclature}

$\begin{array}{ll}\text { AO } & =\text { atomic oxygen } \\ \text { GRC } & =\text { Glenn Research Center } \\ \text { NASA } & =\text { National Aeronautics and Space Administration } \\ \text { NDS } & =\text { NASA Docking System } \\ \sigma & =\text { standard deviation } \\ \text { SPC } & =\text { statistical process control }\end{array}$

\section{Introduction}

STATISTICAL process control (SPC) in conjunction with control charts have long been used as tools to monitor $S$ and control a given process ${ }^{1,2}$. All processes are subject to some form of variability, which if not controlled, can decrease quality, increase cost, and lead to degradation of performance. SPC is crucial in the development and implementation of spaceflight hardware. Due to the inherent increased cost and risk of spaceflight, it is imperative that design specifications and performance requirements be met. More importantly the performance requirements must be repeatable and reliable.

In recent years, the National Aeronautics and Space Administration (NASA) has been developing a low-impact docking system designed for future space exploration vehicles ${ }^{3}$. The low-impact mating operation supports reduction of structural mass and overall mission cost as well as simplifies operations and increases safety ${ }^{4}$. However, this novel system is not without challenges. An integral component of the NASA Docking System (NDS) is the dynamic seal, shown in Fig. 1, located at the main interface between active and passive docking components. This seal separates the habitable environment within the vessel from the exterior vacuum of space and is designed to

\footnotetext{
${ }^{1}$ Research Assistant Professor, College of Engineering, AIAA Member.

${ }^{2}$ Research Associate Professor, Department of Mechanical Engineering, AIAA Senior Member.
} 


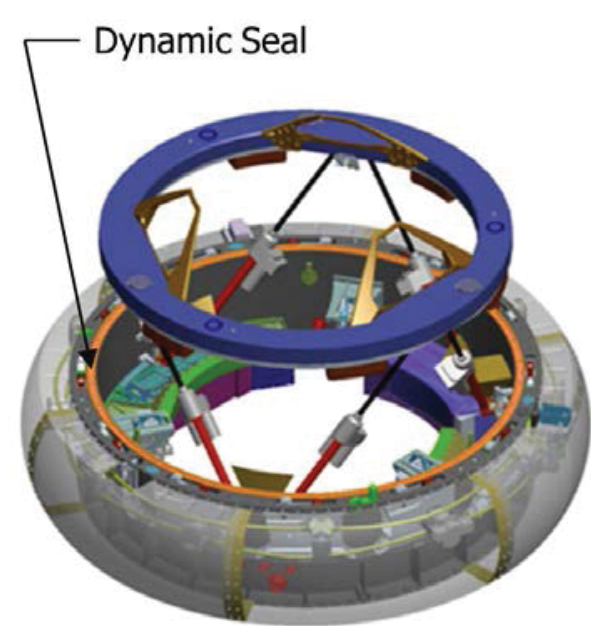

Figure 1. Illustration of the NDS dynamic seal installed on the active docking component. operate in the harsh space environment while performing within strict loading requirements and maintaining an acceptable level of leak rate. A specific requirement is the closure force be within the capabilities of the NDS latching mechanism ${ }^{5}$. If this requirement is not met, the seal will not fully engage and its leak rate may be higher than expected ${ }^{6-9}$ leading to mission failure or catastrophe.

Historically, docking seals have been made of elastomers ${ }^{10}$; silicone elastomers in particular for their operational temperature range and reusability ${ }^{11}$. An added benefit in the case of the NDS is that the material is flexible and of low durometer requiring lower closure (compression) force ${ }^{12}$ and the ability to comply with surface irregularities. Researchers at NASA Glenn Research Center (GRC) have put forth extensive efforts to characterize the mechanical and sealing performance of silicone elastomer docking seals. A variety of subscale candidate docking seals with different geometries, elastomer compounds, operating temperatures, and conditions have been tested ${ }^{12-18}$. The compression force of the seals has been shown to depend greatly on compound ${ }^{12}$, temperature ${ }^{12,16,19,20}$, and cycling $^{16}$ providing useful information for expected performance within an anticipated operational envelope. However, little research has been done to determine repeatability of the compression force when multiple samples of the same design are tested under replicate conditions. Due to the stochastic nature of elastomers, scatter is expected to be observed within the mechanical performance data. To ensure successful seal operation, it is important to understand whether the scatter is due to common or special cause variability as defined in SPC.

The goal of this work was to quantify and characterize the closure force of a custom designed subscale candidate silicone elastomer docking seal. The closure force of multiple test articles was evaluated at room temperature for two complete compression cycles and compared to the NDS latching mechanism requirement. SPC was used to evaluate scatter within the dataset and determine possible sources of variation.

\section{Description of the Experiments}

A series of experiments were performed to quantify and characterize the mechanical performance of a custom designed subscale candidate docking seal. Multiple test articles were manufactured for experimental testing. The compression force required for simulated docking closure was determined for each test article using the experimental setup and procedures detailed herein.

\section{A. Test Article}

The test articles used in this investigation were a custom design consisting of two parts: (1) a molded elastomer seal with primary and redundant seal bulbs and an interconnecting web, and (2) a corresponding two-piece aluminum retainer. The elastomer seal component is referred to herein as the test article. Together the seal and retainers are referred to as the seal assembly. A photograph of a similar seal assembly is shown in Fig. 2. The test articles, manufactured from elastomer compound S0383-70, were molded by the Parker Hannifin Corporation and had a nominal 70 shore A durometer hardness. The two-piece retainer was manufactured from aluminum alloy and anodized for improved corrosion resistance.

A total of 84 subscale test articles were included in this study. For additional testing outside the scope of this work, the test articles were exposed, at NASA GRC's Large Area Atomic Oxygen Exposure Facility, to atomic oxygen (AO) ranging in fluence from 0 to $2.38 \times 10^{20}$ atoms $/ \mathrm{cm}^{2}$. It was assumed that the AO exposure did not affect the results of this study as evidenced in previous work ${ }^{21}$.

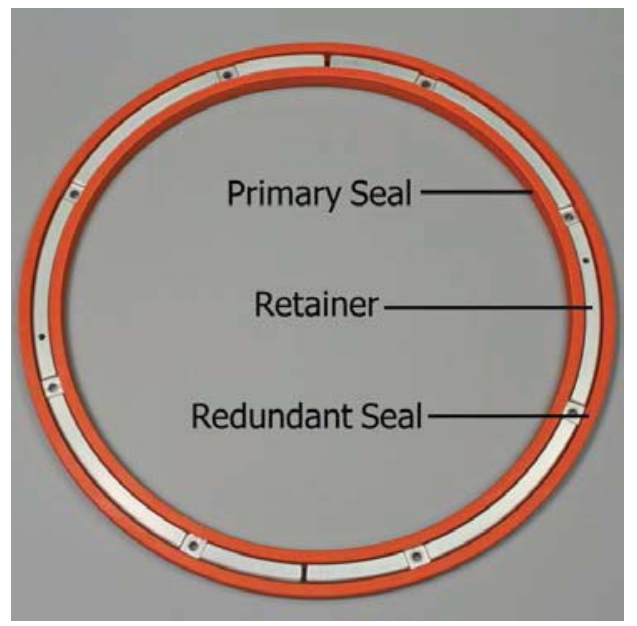

Figure 2. Photograph of a similar seal assembly design. 


\section{B. Seal Assembly Installation}

The test section consisted of two plates machined from aluminum, each with a surface finish better than $0.4 \mu \mathrm{m}$. The upper plate was flat and simulated a seal-to-metal docking interface. The lower plate was grooved to receive the seal assembly and allowed for $18 \%$ compression of the test article's nominal height. During installation, the twopiece retainer was placed between the seal bulbs of the test article, illustrated in Fig. 2, and the seal assembly was installed in the groove of the lower plate. To prevent air entrapment during compression, the test plates were designed with vent holes in the center cavity and between seal bulbs.

\section{Test System and Procedure}

Excluding a subset of nine samples, each test article was compressed twice, in succession. The remaining nine test articles were compressed only once for a total of 159 closure tests. The tests were conducted using an electromechanically actuated test system. This setup, shown schematically in Fig. 3, consisted of the following components: a load frame with actuator rod, a load cell with uncertainty better than $\pm 0.99 \%$ of the reading in

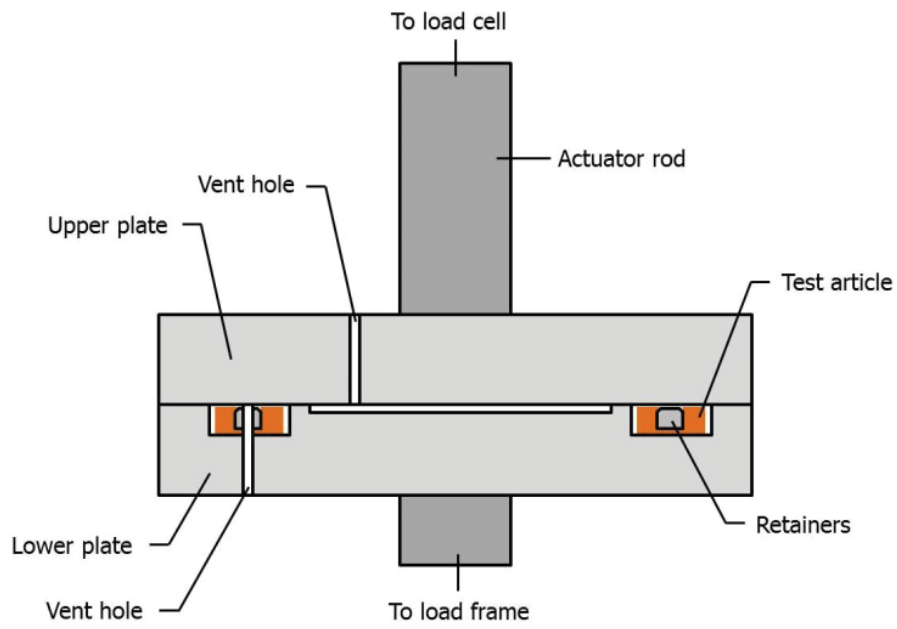

Figure 3. Cross-section of the closure force test section setup. compression, and the two test plates described in the previous section. A laser extensometer was used to measure the displacement between platens. Additionally, a data acquisition system recorded the force response data at a rate of 250 $\mathrm{Hz}$.

During closure tests, the plates complete with seal assembly were installed into the load frame with the upper plate attached to the actuator rod. To simulate docking, the actuator rod was lowered at a nominal rate of 0.2 $\mathrm{mm} / \mathrm{sec}$, until the test article was compressed to a load above $26 \mathrm{kN}$. At a load of this magnitude, plate-to-plate contact was ensured and the force just prior to complete metal-to-metal contact, the closure force, could be determined. The second compression test was run after the seal assembly was uncompressed for a minimum time of eight hours.

\section{Analysis Methodology}

The acquired load versus sample number was used with a visual post-processing methodology ${ }^{12}$ to determine the closure force. The force was plotted on a per data point basis which resulted in three defined regions shown in Fig. 4. These regions were (1) the upper near linear region, (2) the lower near linear region, and (3) the knee of the curve located between the two near linear regions. The knee of the curve was the region where the compression transitioned from elastomer compression to metal-to-metal contact. An ideal test system would consist of a rigid test system with perfectly parallel platens, a level sealing surface, and a homogeneous material that does not exhibit strain hardening. In this case, the transition from the lower linear region to the upper linear region would be marked by a single datum point. This point was represented by the intersection between lines 1 and 2 in Fig. 4 . However, since the experimental test conditions were not ideal and an instantaneous transition from elastomer to metal-tometal contact was not observed, the reported closure force was determined visually.

The data set was parsed such that 100 data points on either side of the visually selected midpoint of the knee were analyzed. Lines were visually fit to the near linear portions of the data curve (lines 1 and 2 in Fig. 4). From this, four different closure force values from least conservative (point D) to most conservative (point A) were obtained. The most conservative value was the force that corresponded to the point at which line 1 deviated from the linear portion of the data set. This point corresponded to the end of the knee of the curve. Similarly, the least conservative value was the force that corresponded to the point at which line 2 deviated from the data set. This was the point that corresponded to the beginning of the knee of the curve. Between the most and least conservative values were two mid-conservative values. The first mid-conservative force (point $\mathrm{C}$ ) was determined by the intersection of lines 1 and 2 (the idealized case). The second mid-conservative force (point B) was determined by the intersection of line 2 with the constructed vertical line drawn from the deviation of line 1 with the data set down to the $\mathrm{x}$-axis. This point represented a repeatable location of higher value than the idealized case; since the near linear 
regions were not perfectly linear, i.e., the strain hardening behavior of the elastomer produced a concave upward bend in the lower near linear region.

The closure force values reported herein were the mid-conservative results established at point $\mathrm{B}$. For the purpose of this paper, the closure force values were normalized by the average compression force value of all 159 tests. The uncertainty of the load cell, better than $\pm 0.99 \%$ as previously mentioned, was considered insignificant and thus the corresponding values were not reported within.

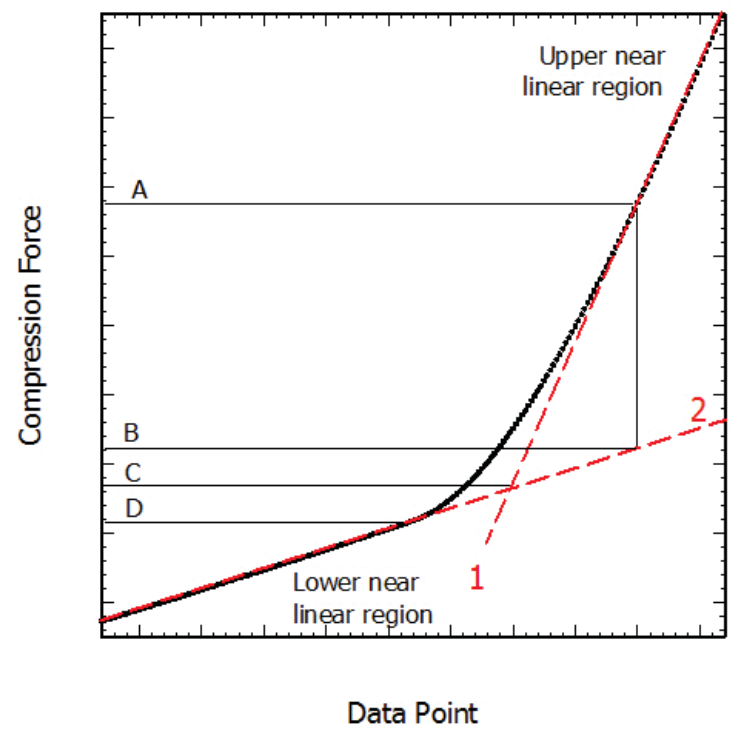

Figure 4. Compression force analysis methodology.

\section{Results and Discussion}

Eighty-four subscale candidate docking seals underwent compression testing at room temperature. For this study room temperature was approximately $23^{\circ} \mathrm{C}$. Each test article was compressed up to two times with a minimum of eight hours at rest, in an uncompressed state, between cycles. The normalized results of these tests are shown in Fig. 5. The dashed line represents the mean of the entire data set, including both first and second cycle compression data.

The compression results, shown in Fig. 5, were consistent with past studies ${ }^{12}$. For a given test article, the first compression cycle resulted in a higher compression force than the subsequent compression cycle. Nevertheless, all resulting compression force values, regardless of cycle, fell below the NDS requirement. This result was not surprising since these tests were performed at room temperature; a relatively mild temperature considering the operational envelope of the silicone elastomer.

Until test article number 63 the closure force was repeatable with a standard deviation of 0.07 and variance of 0.005 for the first cycle. Similarly, the second cycle had a standard deviation of 0.04 and a variance of 0.002 . At test article number 63, the mean value of the compression forces increased approximately $27 \%$ and $21 \%$ for the first and second test cycles, respectively. It was noted that all tests had been performed in the same fashion following established procedures. No remarkable change in test procedures had occurred at the transition

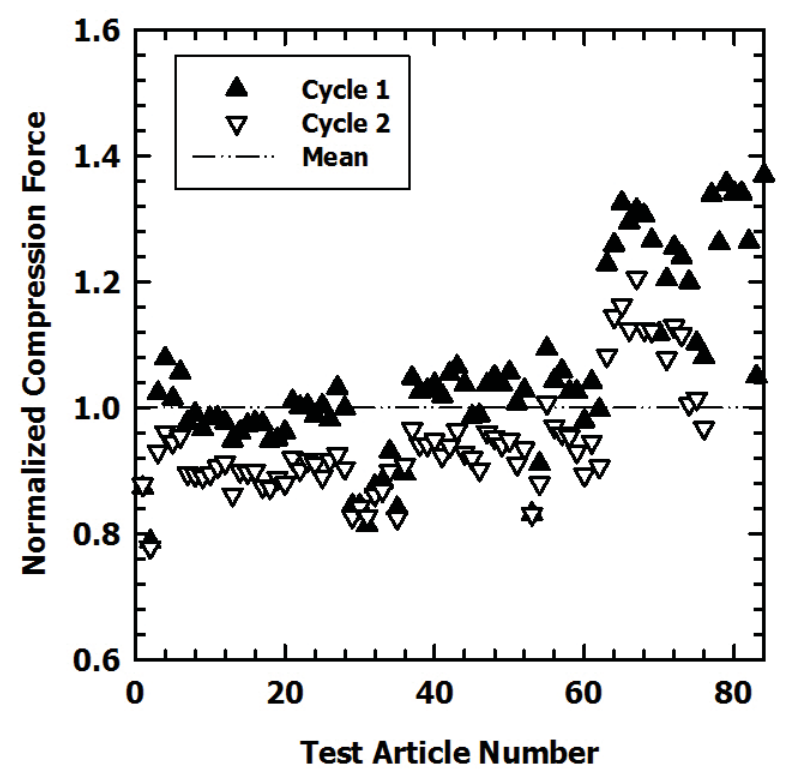

Figure 5. Normalized compression force results from closure tests at room temperature. 
between test articles 62 and 63.

Statistical process control was implemented to further investigate the unanticipated increase in closure force. For each test article, the first and second cycle closure forces were averaged together. So as not to skew the results, the nine tests articles with a single compression cycle were excluded from the SPC analysis. Assuming controlled operation, statistics were run on the first 20 data points to develop a reference data set. The mean, $\pm \sigma, \pm 2 \sigma$, and $\pm 3 \sigma$ of the reference set were plotted together in Fig. 6 with the remaining 55 average closure force data points.

This process control chart in Fig. 6 was used to indicate areas of possible process change during testing or manufacturing. A lack of process control was indicated by the following scenarios: (1) one or more data points beyond $\pm 3 \sigma$, two of three data points beyond $\pm 2 \sigma$, (3) four of five data points beyond $\pm \sigma$, and/or (4) eight consecutive data points on the same side of the average ${ }^{1,2}$. Areas with a potential lack of process control are circled in Fig. 7 with the worst violations highlighted in red. Not surprisingly, a significant lack of process control occurred at test article 63 and beyond. Since these data points fell outside the $+3 \sigma$ limit the variability was likely caused by special circumstances. Still, potential less significant variations occurred throughout the entire data set; because the closure tests were run following established test procedures in a controlled environment, these inconsistencies were likely the result of common cause variability.

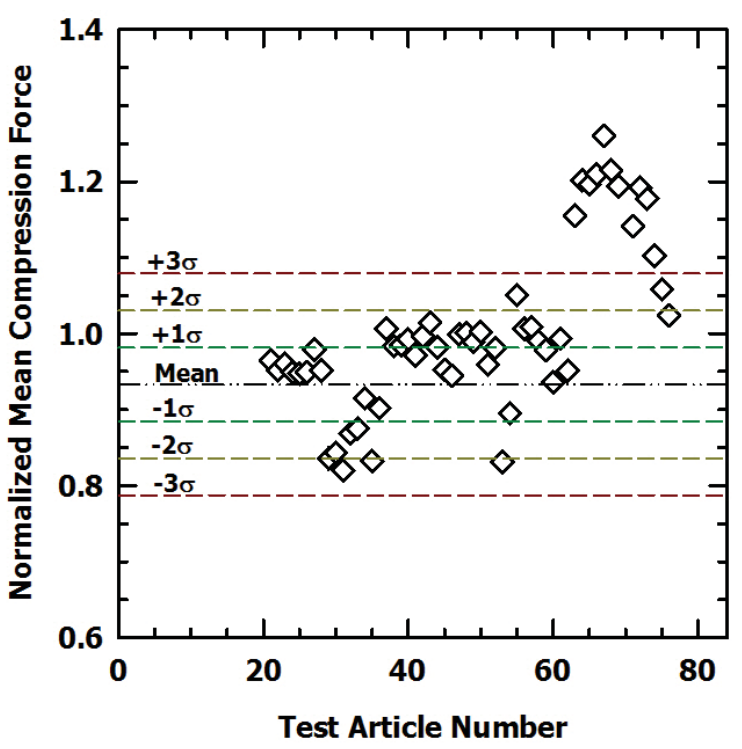

Figure 6. Statistical process control chart for average closure force values.

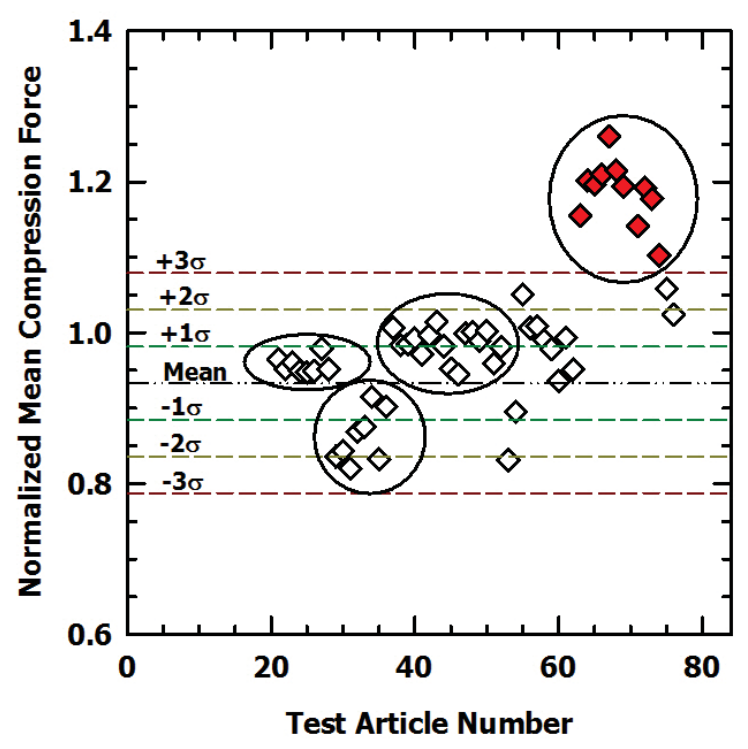

Figure 7. Areas of potential lack of process control.

Potential sources of special cause variation were explored. Nothing of note had changed between the test procedures implemented for test articles 62 and 63. Though, it was discovered that the molding process of the test articles had been modified after the first 62 seals had been manufactured. The first 62 test articles were manufactured in bulk and were prepared in a single cavity subscale seal mold. The remaining seals were manufactured at a later time in a multi-cavity subscale seal mold.

To further investigate the elevated closure forces, type $\mathrm{M}$ durometer tests to determine the material hardness were completed on test articles that were manufactured in each type of mold. Two samples from the single cavity mold and four samples from the multi-cavity mold were tested following ASTM standards ${ }^{22}$. The mean type M durometer hardness was $6 \%$ higher for the multi-cavity molded test articles than for the single cavity molded test articles. A student's t-test was implemented to compare the means of the two groups. With $95 \%$ confidence, there was a statistically significant difference between the mean values of the two groups. This, coupled with the results of the closure force tests suggested a variation during manufacturing rather than testing. Since the test articles were not manufactured in-house it was difficult to pinpoint the source. Whether it was due to mold change, material batch change, or alterations in the curing process, further investigation is needed to determine the source of variation. 


\section{Conclusions}

NASA has been developing a novel docking system for future space missions. This docking system is designed with the unique capability to attenuate high-impact docking loads relieving system complexity and improving mission safety. An integral component of the docking system, the dynamic seal, serves as the gas barrier between the interior of the spacecraft and the external vacuum of space. For safety and success of future manned spaceflight missions, this seal must perform within strict requirements, including closure force.

This study focused on the quantification and characterization of the mechanical performance of a subscale candidate silicone elastomer docking seal design tested under replicate conditions. Eighty-four test articles were compression tested at room temperature to determine the closure force of the elastomer seal. The results were within the requirements of the NDS latching mechanism, but a significant amount of scatter was observed, especially for those test articles from number 63 to 84 . A statistical process control chart indicated possible lack of control during manufacturing. Additional type $\mathrm{M}$ durometer tests supported these findings. A change in the manufacturing molding process was noted, but whether the process variation was due to mold change, elastomer batch change, alterations made to the curing process, and/or any other manufacturing change was difficult to resolve. Further investigation is needed to determine the exact source of variation. However, knowledge that a seemingly insignificant process change could alter the expected performance of a candidate docking seal is crucial. It leads to improved development and process control of spaceflight hardware and ultimately to the success and safety of future spaceflight missions.

\section{Acknowledgments}

The authors acknowledge Shawn C. Taylor of the University of Toledo and Janice L. Wasowski of The University of Akron for their contribution to this work. Support for this work was provided by the National Aeronautics and Space Administration under contract NNC08CA35C.

\section{References}

${ }^{1}$ Scheaffer, R. and McClave, J., Probability and Statistics for Engineers, Duxbury Press, Belmont, California, 4th ed., 1995.

${ }^{2}$ Box, G., Hunter, J., and Hunter, W., Statistics for Experimenters: Design, Innovation, and Discovery, John Wiley \& Sons, Inc., Hoboken, New Jersey, 2nd ed., 2005.

${ }^{3}$ Lewis, J. L., Carroll, M. B., Morales, R. H., Thang, D. L., and NASA, Washington, D. C., Androgynous, Reconfigurable Closed Loop Feedback Controlled Low Impact Docking System with Load Sensing Electromagnetic Capture Ring, United States of America Patent No. 6,354,540, 12 March 2002.

${ }^{4}$ LaBauve, T., Low-impact Docking System, Biennial Research and Technology Development Report: Johnson Space Center, edited by H. Lane and K. Lulla, No. TM-2009-214795, Johnson Space Center, Dec 2009, pp. 196-197.

${ }^{5}$ International Space Station Multilateral Coordination Board, International Docking System Standard (IDSS) Interface Definition Document (IDD), Tech. Rep. Revision C, 20 November 2013.

${ }^{6}$ Smith, I. M., Daniels, C. C., Dunlap, Jr., P. H., and Steinetz, B. M., Performance of Sub-scale Docking Seals under Simulated Temperature Conditions, Proceedings of the 44th AIAA / ASME / SAE / ASEE Joint Propulsion Conference \& Exhibit, No. AIAA 2008-4713, Hartford, CT, 21-23 July 2008.

${ }^{7}$ Wasowski, J. L., Penney, N., Garafolo, N. G., and Daniels, C. C., Leak Rates of a Candidate Main Interface Seal at Selected Temperatures, Proceedings of the 45th AIAA / ASME / SAE / ASEE Joint Propulsion Conference \& Exhibit, No. AIAA 20095320, 2009.

${ }^{8}$ Garafolo, N. G. and Sawyer, S. D., A Computational Prediction of the Permeation Leak Rate Performance of a Candidate Space Seal, Proceedings of the 49th AIAA Aerospace Sciences Meeting, No. AIAA 2011-425, Orlando, Florida, 4-7 January 2011.

${ }^{9}$ Garafolo, N. G. and Daniels, C. C., Experimental Investigation of Leak Rate Performance of a Subscale Composite Elastomer-Retainer Docking Seal, Journal of Spacecraft and Rockets, Vol. 50, No. 3, 2013, pp. 709-714.

${ }^{10}$ Finkbeiner, J. R., Dunlap, Jr., P. H., Steinetz, B. M., and Daniels, C. C., Review of Seal Designs on the Apollo Spacecraft, Journal of Spacecraft and Rockets, Vol. 45, No. 5, September - October 2008, pp. 900-910.

${ }^{11}$ Parker Hannifin Corporation, Parker O-Ring Handbook, Parker Hannifin Corporation, Cleveland, Ohio, 2001.

${ }^{12}$ Oravec, H. A., Panickar, M. B., Wasowski, J. L., and Daniels, C. C., Influence of Elastomer Compound and Test Temperature on the Compression Force of Candidate Space Seals: A Preliminary Study, Proceedings of the 47th AIAA / ASME / SAE / ASEE Joint Propulsion Conference \& Exhibit, No. AIAA 2011-5709, AIAA, San Diego, CA, 31 July-3 August 2011.

${ }^{13}$ Daniels, C. C., de Groh III, H., Dunlap, Jr., P. H., Finkbeiner, J. R., Steinetz, B. M., Bastrzyk, M. B., Oswald, J. J., Banks, B. A., Dever, J. A., Miller, S. K., and Waters, D. L., Characteristics of Elastomer Seals Exposed to Space Environments, Proceedings of the 43rd AIAA / ASME / SAE / ASEE Joint Propulsion Conference \& Exhibit, No. AIAA 2007-5741, Cincinnati, OH, 8-11 July 2007. 
${ }^{14}$ Daniels, C. C., Oswald, J. J., Bastrzyk, M. B., Smith, I. M., Dunlap, Jr., P.H., and Steinetz, B. M., Experimental Investigation of Elastomer Docking Seal Compression Set, Adhesion, and Leakage, Proceedings of the AIAA Space 2007 Conference and Exposition, No. AIAA-2007-6197, Long Beach, CA, 18-20 September 2007.

${ }^{15}$ Garafolo, N. G. and Daniels, C. C., An Experimental Investigation of the Leak Rate Performance of a Subscale Candidate Elastomer Candidate Docking Seal, Proceedings of the 46th AIAA / ASME / SAE / ASEE Joint Propulsion Conference \& Exhibit, No. AIAA 2010-6907, 2010.

${ }^{16}$ Bastrzyk, M. B. and Daniels, C. C., The Mechanical Performance of Subscale Candidate Elastomer Docking Seals, Proceedings of the 51st AIAA Materials Conference, No. AIAA 2010-3129, AIAA, Orlando, FL, 12-15 April 2010.

${ }^{17}$ Hartzler, B. D., Panickar, M. B., and Daniels, C. C., Comparison of Adhesion and Retention Forces for Two Candidate Docking Seal Elastomers, Proceedings of the 52nd AIAA/ASME/ASCE/AHS/ASC Structures, Structural Dynamics and Materials Conference, No. AIAA 2011-2158, AIAA, Denver, Colorado, 4-7 April 2011.

${ }^{18}$ Dunlap, Jr., P. H., Martin, R. E., Garafolo, N. G., Oravec, H. A., and Steinetz, B. M., Durability Testing of Docking System Seals for Space Applications, Proceedings of the 47th AIAA / ASME / SAE / ASEE Joint Propulsion Conference \& Exhibit, No. AIAA 2011-5710, AIAA, San Diego, CA, 31 July-3 August 2011.

${ }^{19}$ Bastrzyk, M. B., Garafolo, N. G., and Daniels, C. C., Compression Force Response and Leak Rate Quantification of Candidate Static Silicone Space Seals, Proceedings of the 46th AIAA Joint Propulsion Conference \& Exhibit, No. AIAA 20106908, AIAA, Nashville, TN, 25-28 July 2010.

${ }^{20}$ Daniels, C. C., Garafolo, N. G., Bastrzyk, M. B., and Smith, I. M., Evaluation of a Novel Seal for Space Applications, Journal of Spacecraft and Rockets, Vol. 49, No. 1, 2012, pp. 83-90.

${ }^{21}$ Garafolo, N. G., Bastrzyk, M. B., and Daniels, C. C., The Effects of Atomic Oxygen on the Sealing and Mechanical Performance of an Elastomer Seal, Proceedings of the 48th AIAA Aerospace Sciences Meeting, No. AIAA 2010-1440, 2010.

${ }^{22}$ Standard Test Method for Rubber Property - Durometer Hardness, Standard D 2240-05, ASTM International, West Conshohocken, Pennsylvania, 2005. 